\title{
Metodología de la enseñanza centrada en el aprendizaje
}

Ma. Dolores Villalpando Calderón

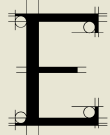

$n$ el proceso educativo escolar, la función del profesor es la enseñanza, y la del estudiante es la realización de acciones que conduzcan al aprendizaje. Los enfoques actuales de la educación plantean procedimientos centrados en el aprendizaje de los estudiantes para conducirlos a la construcción del conocimiento y al logro de competencias sociales, profesionales y laborales, entre otras. En este artículo, se presentan algunos elementos que permiten identificar procedimientos de enseñanza para actualizar la labor docente y facilitar el aprendizaje de los estudiantes dirigido a la formación integral de la persona, como se propone en el Modelo Educativo Institucional de la Universidad Autónoma de Aguascalientes (UAA).

\section{Metodología de la enseñanza}

Para situarnos en el tema de la metodología de la enseñanza, primeramente se hace referencia al término metodología, de manera general y práctica, considerándola como un procedimiento sistemático que conduce a la planeación y realización de acciones para el logro de los objetivos establecidos previamente. Rajadaell concibe la metodología de la enseñanza como "aquella opción que toma el docente o el formador para organizar el proceso de enseñanza - aprendizaje, teniendo presentes una serie de factores que condicionan dicha actuación" (Rajadaell, 2001:1). Es así que la metodología de la enseñanza implica la selección de procedimientos que ha de realizar el profesor para orientar a los estudiantes al logro de aprendizajes.

Considerar a la metodología de la enseñanza como un elemento importante en la práctica docente, implica reflexionar acerca de los procedimientos utilizados en las propias actividades de enseñanza y los resultados que se observan en los aprendizajes adquiridos por los estudiantes. Mi experiencia personal, en el desarrollo de propuestas metodológicas centradas en el aprendizaje de los estudiantes, se fue construyendo en la misma práctica docente durante los años de trabajo en las aulas universitarias, con la reflexión y revisión a partir de teorías cognitivas, ya que una preocupación frecuente siempre ha sido cómo hacer para facilitar el aprendizaje de los estudiantes, tanto de aquellas materias de contenido teórico como de los cursos prácticos donde se requiere centrar la atención en el desarrollo de habilidades.

En 2006, la UAA aprueba su Modelo Educativo Institucional con enfoque constructivista y con la tendencia de un trabajo educativo a 
partir de estrategias centradas en el aprendizaje de los estudiantes. Recientemente, se incursiona en el campo de las competencias académicas y las propias de cada profesión de acuerdo con el área disciplinar. De esta forma, he logrado concretar actividades que desde el principio de los años noventa, venía construyendo con procedimientos estratégicos fundamentados en el enfoque constructivista. Actualmente, continúo realizando la función docente con estudiantes de diferentes carreras y semestres a partir de metodologías y estrategias de enseñanza centradas en el aprendizaje.

Para comprender y fundamentar la función docente y las metodologías orientadas hacia el aprendizaje, es pertinente recurrir a las teorías constructivistas que presentan elementos explicativos relacionados con los procesos intelectuales y la construcción del conocimiento, además de que proponen estrategias cognitivas y metacognitivas, tanto para la enseñanza como para el aprendizaje; las primeras hacen referencia a los procesos que conducen a la construcción de conocimiento y las segundas permiten la reflexión y concientización de los conocimientos y competencias adquiridas, así como los procedimientos requeridos para su construcción.

Coll (en Díaz Barriga y Hernández, 2005:28) presenta, desde diferentes teorías, los elementos explicativos acerca de cómo el aprendiz construye sus conocimientos; él señala que "la postura constructivista en educación se alimenta de las aportaciones de diversas corrientes psicológicas: el enfoque psicogenético piagetano, la teoría de los esquemas cognitivos, la teoría ausubeliana del aprendizaje significativo, la psicología social vigotskiana, que comparten el principio de la importancia de la actividad constructiva del alumno en la realización de los 
aprendizajes escolares". Esta postura considera que los sujetos construyen el conocimiento activamente, no lo reciben pasivamente, como ocurre en la práctica de enseñanza centrada en la exposición del docente, sino que la función del profesor es de facilitador del aprendizaje, y su objetivo: "no consiste en proporcionar información a sus alumnos, sino en conseguir que piensen" (Nickerson, Perkins y Smith, 1998:12).

Con esta breve explicación del enfoque constructivista, se puede entender por qué el Modelo Educativo Institucional (2006), fundamentado en estos principios, indica que en la formación intelectual, los estudiantes generarán procesos básicos y superiores de pensamiento y serán capaces de construir aprendizajes significativos y útiles para solucionar problemas de la vida profesional, laboral y cotidiana, de forma original y creativa. Por lo tanto, señala que el aprendizaje es la finalidad de la enseñanza y un proceso de construcción del conocimiento, aspirando a un aprendizaje de calidad, integral y centrado en el alumno, promoviendo el aprendizaje significativo mediante la recuperación de los conocimientos previos.

Para diseñar una metodología de enseñanza "se necesita planificar estrategias que aproximen al máximo hacia la obtención de las finalidades previstas, a través de actividades concretas, activas y graduales, y con el soporte de materiales curriculares, así como el espacio y el tiempo más adecuados a cada estrategia" (Rajadaell, 2001:1). Considerando los elementos de esta postura teórica del aprendizaje, se presentan algunas sugerencias para construir metodologías, esperando que sean de utilidad para los profesores de educación media superior y superior que deseen optimizar su función docente en beneficio de los estudiantes.

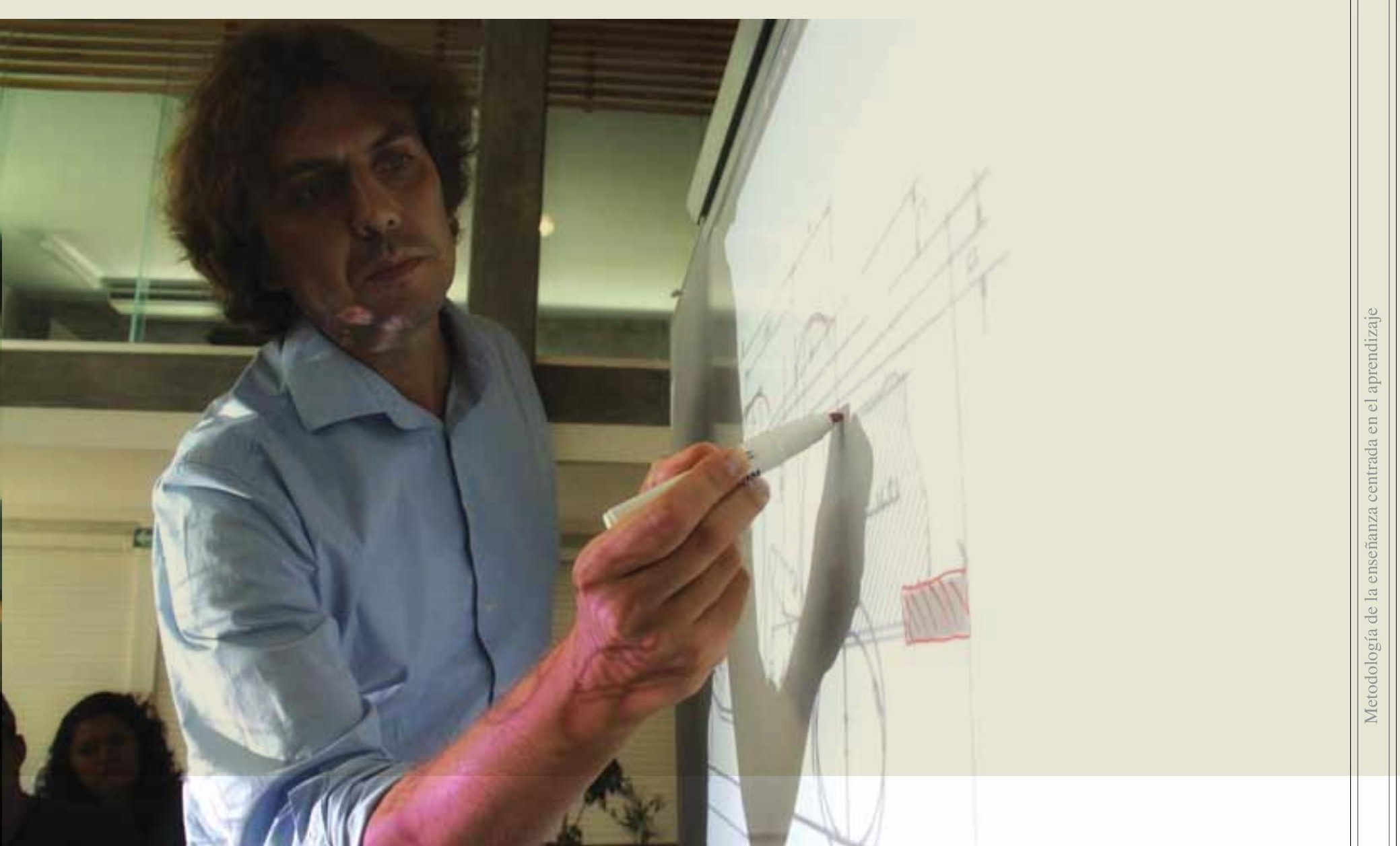


Diseño de una metodología de la enseñanza
centrada en el aprendizaje

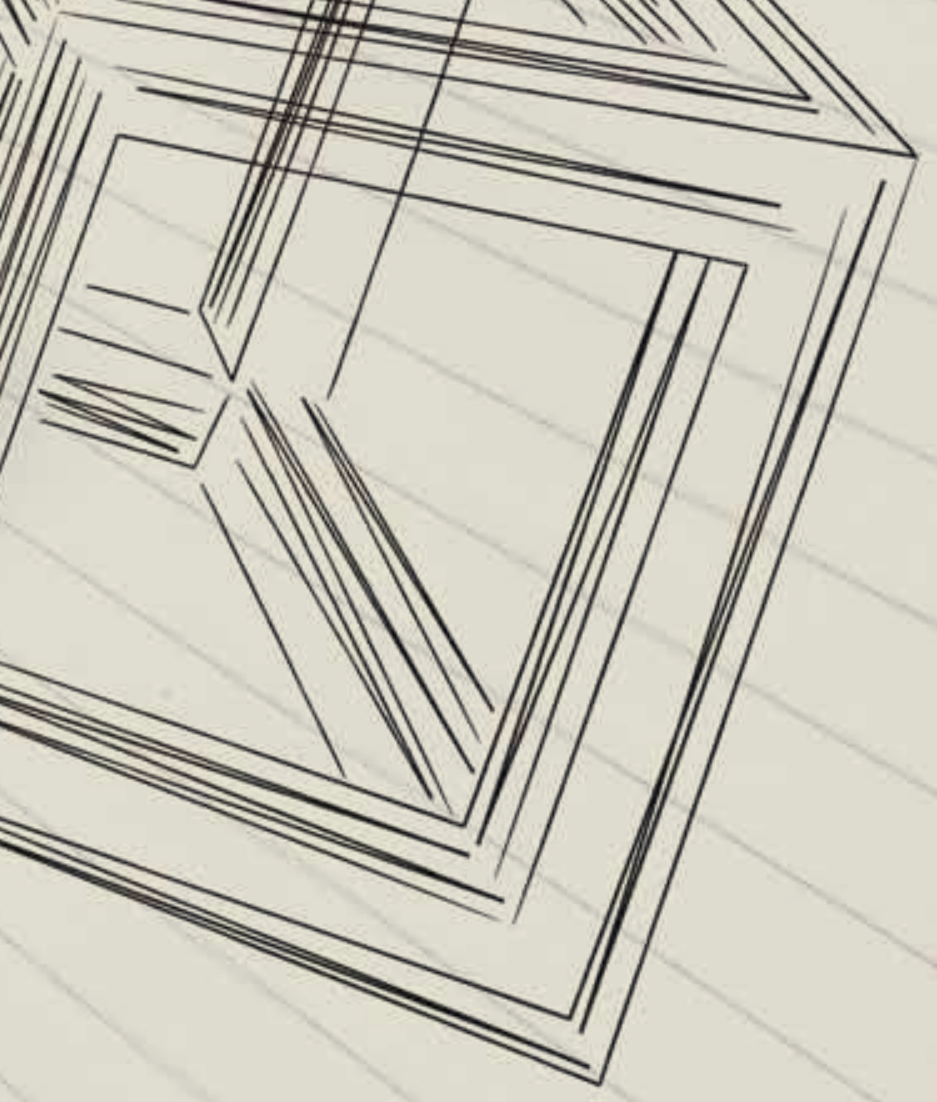


El diseño de metodologías de enseñanza para la práctica docente "es una competencia importante del profesor" (Estévez, 2004:20), que requiere de un conjunto de elementos para estructurar procedimientos estratégicos que conduzcan las actividades del profesor para facilitar el aprendizaje del alumno a partir de la construcción del conocimiento, el desarrollo de habilidades y la promoción de actitudes y valores:

1 Identificar las características del grupo (edad, nivel escolar, desarrollo intelectual, entre otras) detectando las necesidades educativas de los estudiantes.

20 Consultar las propuestas de los programas de desarrollo y modelos educativos nacionales e institucionales para identificar los fines y tendencias educativas.

$3^{\mathrm{O}}$ Fundamentar el diseño metodológico a partir de los principios, elementos y explicaciones de las teorías del enfoque constructivista que sustenten las actividades y estrategias propuestas para los procedimientos de la enseñanza centrada en el aprendizaje.

$4^{\mathrm{O}}$ Formular los objetivos de acuerdo con las taxonomías de enfoque constructivista y conforme a los procesos del pensamiento que se involucrarán en el proceso de aprendizaje (observación, comparación, relación, clasificación, ordenamiento, análisis, síntesis, evaluación, procesos analógicos, hipotéticos, inductivos, deductivos, etcétera).

$5^{\mathrm{O}}$ Seleccionar los contenidos del programa de la materia correspondiente que se desarrollará durante el período escolar.

$6^{0}$ Seleccionar las actividades del docente y las que ha de realizar el estudiante para la construcción del conocimiento, las habilidades y la promoción de actitudes y valores.

$7^{\mathrm{O}}$ Seleccionar, adaptar o diseñar estrategias en donde se propongan los procedimientos a seguir tanto para la realización de la función del docente como del estudiante y para el logro del aprendizaje de contenidos declarativos (conocimiento), procedimentales (procedimientos) y actitudinales (actitudes y valores) de acuerdo con los objetivos propuestos.

- Las estrategias se auxilian de los procedimientos generales que ofrecen los diferentes métodos de enseñanza, los procedimientos más específicos de las técnicas y de los recursos materiales y humanos necesarios para llevar a cabo las actividades propuestas.

- En el diseño de las estrategias se integran los elementos anteriores: los procesos de pensamiento, los contenidos, los procedimientos y los criterios de evaluación de los aprendizajes para cada sesión de acuerdo con los objetivos y el tema a tratar. 
- Se recomienda que las actividades propuestas para el aprendizaje cuenten con guías de trabajo, que contengan instrucciones claras y propongan o sugieran la utilización de materiales o fuentes de información, para orientar de forma segura a los estudiantes hacia la búsqueda de información o la realización de acciones pertinentes, para el desarrollo de las habilidades propuestas en los programas educativos y el logro de la construcción del conocimiento.

$8^{\mathrm{O}}$ Realizar procedimientos de evaluación:

- Diagnóstica o inicial: al inicio del curso para identificar conocimientos previos.

- Formativa o intermedia: durante el desarrollo del curso para ir verificando el logro de objetivos.

- Sumativa o final: al término del curso para verificar el logro del objetivo general.

A partir de los resultados obtenidos en las evaluaciones del aprendizaje de los estudiantes, el profesor habrá de realizar reflexiones acerca de la planeación de las actividades de enseñanza y del propio trabajo docente, lo que permitirá que estas actividades se planeen cada vez con mayor nivel de conciencia y organización.

Fuentes de consulta

Coll, C. y cols. (2007). El Constructivismo en el Aula (17 ${ }^{\mathrm{a}}$. Edición), España: Graó.

Díaz Barriga, Frida y Hernández Rojas, Gerardo (2005). Estrategias Docentes para un Aprendizaje Significativo. Una interpretación constructivista $\left(2^{\mathrm{a}}\right.$. Edición). México: Mc Graw Hill.

Estévez, E. H. (2004). Enseñar a aprender. Estrategias cognitivas. México: Paidós.

Nikerson, R. S., Perkins, D. N. y Smith, E. E. (1998). Enseñar a Pensar. Aspectos de la aptitud intelectual. México: Paidós.

Rajadaell, N. Los procesos formativos en el aula: Estrategias de EnseñanzaAprendizaje. En Sepúlveda, F. y Rajadell, N. (Coords.) (2001). Didáctica General para Psicopedagogos. Madrid: UNED.

Universidad Autónoma de Aguascalientes (2007). Modelo Educativo Institucional. En Correo Universitario, sexta época, núm. 16, publicado el 15 de marzo de 2007. México: UAA. 